\title{
A Grid-enabled Control Plane Architecture: The PHOSPHORUS approach
}

\author{
D. Simeonidou ${ }^{1}$, E. Escalona ${ }^{1}$, G. Zervas ${ }^{1}$, R. Nejabati ${ }^{1}$, S. Spadaro ${ }^{2}$, A. Binczewski ${ }^{3}$, G. Carrozzo ${ }^{4}$, N. Ciulli $^{4}$ \\ ${ }^{I}$ Photonic Network Research Lab, Department of Computing and Electronic Systems, University of Essex, UK \\ ${ }^{2}$ Optical Communications Group, TSC Dept, Universitat Politècnica de Catalunya, Spain \\ ${ }^{3}$ Poznan Supercomputing and Networking Center, Poland \\ ${ }^{4}$ neXtworks, Italy \\ \{dsimeo,eescal,gzerva,rnejab\}@essex.ac.uk,spadaro@tsc.upc.edu,artur@man.poznan.pl, \{g.carrozzo,n.ciulli\}@nextworks.it
}

\begin{abstract}
This paper presents the architecture of the Phosphorus Grid-enabled GMPLS $\left(G^{2}\right.$ MPLS) Network Control Plane that implements the concept of Grid Network Services provisioning through a set of seamlessly integrated procedures. (C)2007 Optical Society of America OCIS codes: (060.4250) Networks; (060.4253) Networks, circuit-switched
\end{abstract}

\section{Introduction}

A new generation of e-Science and Grid applications is emerging that couples scientific instruments, distributed data and high-end computing resources, often interconnected via high-speed optical networks. These applications have requirements for determinism (guaranteed QoS), shared data spaces, large transfer of data and specific latency that are often achievable only through dedicated optical bandwidth (lambdas). With the availability of huge amount of optical bandwidth, Grid networks often use the underlying optical infrastructure opaquely taking the network for granted without any bandwidth reservation for their applications. However, Grid's collaborative and shared nature is ever-scaling in terms of type and number of participating sites, drives the need for a dynamic transport network able to manage and coordinate seamlessly both network and non-network (CPU, storage, etc.) resources. To achieve this, the design and definition of new architectures that implement the concept of Grid Network Services (GNS) is crucial in order to bring closer Grid and network services.

This paper presents one of the main outcomes of the Phosphorus project which is to define and build a new Network Control Plane (NCP) architecture that integrates a GNS provisioning mechanism and allows a single-step co-allocation and reservation of network and Grid computing resources [1]. The aim of NCP is to facilitate dynamic and automatic resource provisioning, including protocols and mechanisms to disseminate resource information. In this paper the relevant requirements and challenges to evolve into an integrated Grid-Network architecture that guarantees Grid and network service provisioning are discussed and Phosphorus solutions are presented.

\section{Photonic Grid Networks Requirements}

The implementation of GNS (i.e., services that allow the provisioning of network and Grid resources in a single-step through a set of seamlessly integrated procedures) poses a number of requirements on the Control Plane. This includes:

- Network and Grid service/resource discovery: the NCP must provide mechanisms for learning and advertisement of the Grid and network resource availability/capability at the Grid user sites. This is referred as GNS neighbor discovery mechanism and must include both network resources (e.g., amount of bandwidth, connectivity, etc.) and Grid resources (e.g., amount of CPU, storage, etc.).

- Connection management: should mainly support Switched Connections (SC) through the G.OUNI (Grid Optical User-Network Interface) [2]. G.OUNI is further described in Section 4. Other solutions made available by ASON/GMPLS architecture, like Soft Permanent Connections (SPC) and Permanent Connections (PC) [3], are not essential for the Photonic Grid Network.

- Flexible bandwidth allocation and advance reservation: they are required to support the dynamicity of Grid jobs and the necessity of guaranteed services at the time of execution of the job [4].

- Service resilience: recovery procedures should provide means for faulty condition detection and reaction, as well as mechanisms for diverse routing between the failing path and its backups when the execution of a task might be compromised by some possible faults of the involved network or Grid resources.

- Photonic Grid Network AAA: mechanisms to provide authentication, authorization and accounting for the service usage are required in the Control Plane scope to integrate AAA infrastructures network and nonnetwork resources, towards a unified and generalized infrastructure granting access to both Grid and network resources. 


\section{Grid-aware GMPLS (G' ${ }^{2}$ MPLS) Control Plane: Phosphorus Control Plane}

The Phosphorus framework identifies different layering solutions with respect Grid Service layer and Network Control Plane positioning. Layers involved are: Grid Service Layer, Network Control Plane and Transport Plane (TP). The Grid Service Layer comprises Grid users, Grid resources, Grid applications and Grid middleware. The NCP takes different roles depending on the architectural model chosen from network configuration to Grid and network resource co-allocation. In Phosphorus, the NCP is called $\mathrm{G}^{2}$ MPLS which is a Grid-aware GMPLS. The $\mathrm{G}^{2}$ MPLS architecture exposes interfaces specific for Grids and is made of a set of extensions to the standard ASON/GMPLS architecture to implement the concept of GNS. Therefore, G ${ }^{2}$ MPLS results in a more powerful NCP solution than the standard ASON/GMPLS, because it will fulfill the needs for enhanced Grid services. Finally, the Transport Plane is the basic layer comprising all the data bearing equipments and their configuration interfaces.

The functionalities associated to each layer and the relationships between them constitute the different Phosphorus architectural models, namely Overlay and Integrated. Note that despite this denomination is equal to the ones given to the IETF GMPLS deployment models [5], the scope differs as IETF is based on relationships between different Control Plane segments/domains and $G^{2}$ MPLS refers principally to the positioning between the Grid Service Layer and the NCP. Both $\mathrm{G}^{2}$ MPLS Overlay and Integrated models share the same goals; the difference lies on the layering solutions adopted.

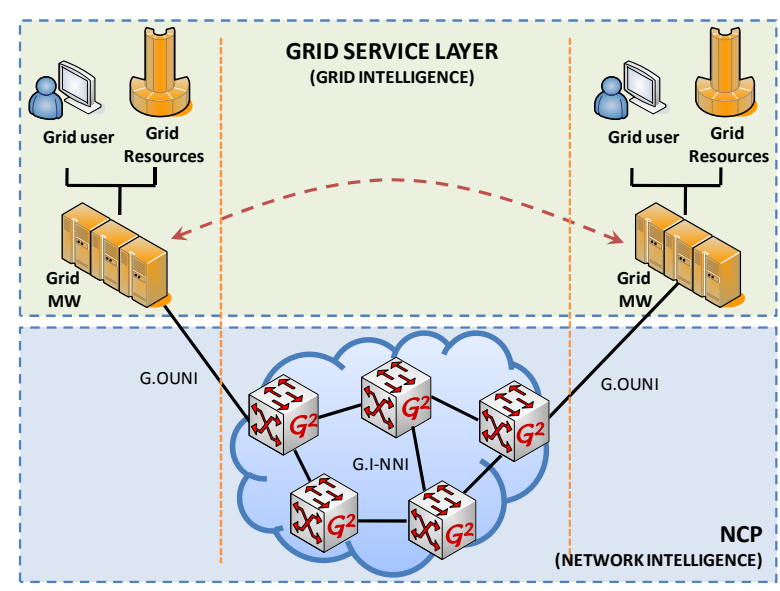

(a)

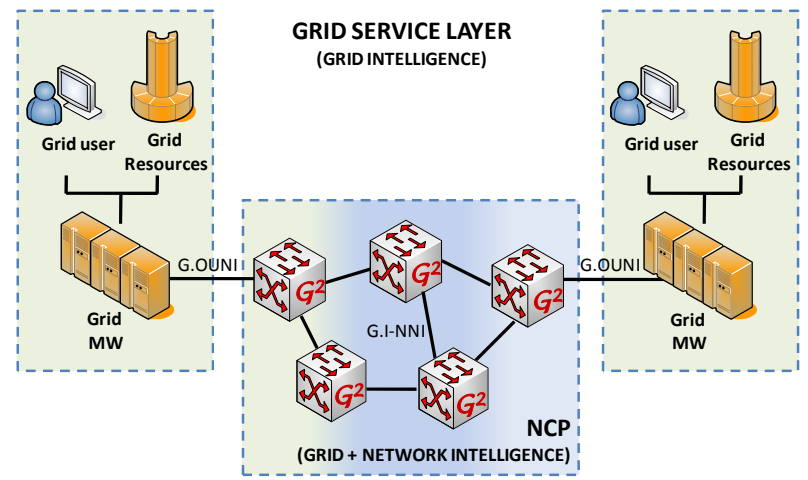

(b)

Fig. 1: $\mathrm{G}^{2}$ MPLS Architectures. (a) Overlay Model and (b) Integrated Model

In the $G^{2}$ MPLS Overlay model (Fig. 1a), computational and service intelligence is maintained on the Grid layer and, upon the occurrence of a Grid job request from a user, it is up to the Grid scheduler to initiate and coordinate the reservation process. All the discovery and co-allocation information regarding Grid resources is managed by the Grid layer in order to provide Grid resource configuration and monitoring as well as Grid resource scheduling services. On the other hand, the NCP is responsible for scheduling, configuring and implementing advance reservations, recovery and connection monitoring mechanisms to meet job networking requests.

In the $\mathrm{G}^{2}$ MPLS Integrated model (Fig. 1b), most of the functionalities akin to the Grid Service Layer for Grid resources such as localization or commit of the advance reservations are moved to the Network Control Plane which will provide scheduling, configuration and co-allocation of remote Grid and network resources. However, each involved Grid local site is still responsible for configuration and scheduling of local resources. For resource localization purposes, Grid sites are considered as special network nodes with specific additional Grid resource information and are added to the network topology. Grid middleware/applications specify the destination Grid site or declare an anonymous service requirement and leave the NCP the responsibility of allocating the best resource candidate for that service.

\section{4. $G^{2}$ MPLS Network Reference Points}

The deployment of the enhanced $G^{2}$ MPLS Control Plane sets analogous reference points with respect to the ones defined by ASON/GMPLS [5]. The resulting network interfaces are a Grid-aware evolution of the standard interfaces (UNI, I-NNI, E-NNI), with a set of procedures that maintains the backward compatibility with the original ASON references, but provides also the seamless and one-step control of both Grid and network resources. 
Five network interfaces are identified in the $\mathrm{G}^{2}$ MPLS Network Control Plane:

- G.OUNI: interface between Grid site/user and the NCP that enables interoperable procedures to facilitate on demand as well as in-advance Grid and network services over the NCP [2]. G.OUNI supports connectivity service through the transport network for Grid end-points, signaling to invoke Grid and network services and auto-discovery procedures to aid signaling. Under the overlay model, G.OUNI basically offers network services, but information about Grid resource capability and availability has to be exchanged between middleware modules using the NCP as no IP layer interconnects them. Under the integrated model, G.OUNI also supports meta-scheduling processing previously operated by the Grid middleware and now provided at the NCP level, including Grid service/resource discovery, advance reservation service and co-allocation.

- G.I-NNI: interface that supports routing and signaling procedures between $\mathrm{G}^{2}$ MPLS adjacent nodes. It has knowledge of the GNS semantic and implements the mechanisms for the creation, deletion, monitoring, recovery and crankback of the network connections between neighboring nodes.

- G.E-NNI: interface that propagates Grid and network topology information across different CP domains. It has also knowledge of the GNS semantic and implements the inter-domain mechanisms for the creation, deletion, monitoring, recovery and crankback of network connections across domains.

- SBI: interface between NCP and TP that provides specific network resource configuration.

- NBI: interface between NCP and the Grid Service Layer providing resource indexing, selection and reservation.

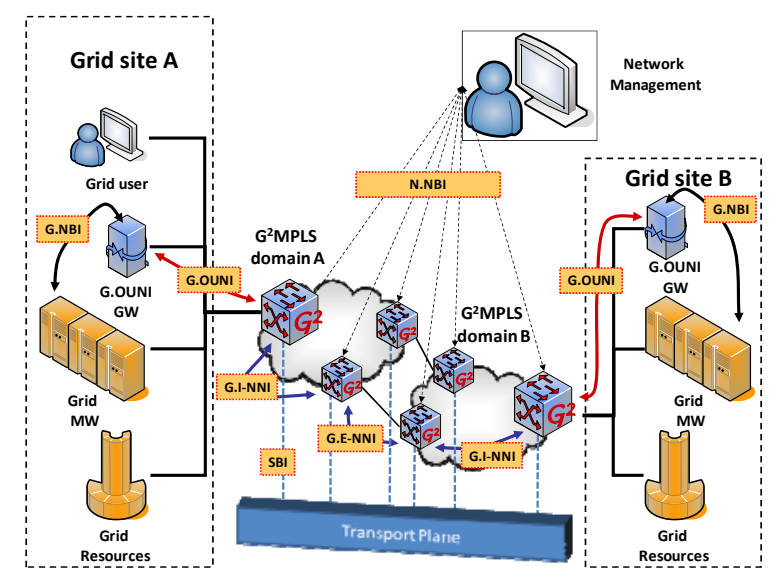

Fig. 2: $\mathrm{G}^{2}$ MPLS Reference Points

In Fig. 2 all these reference points are represented in an example scenario including two neighboring $\mathrm{G}^{2}$ MPLS domains, interfaced through G.OUNI gateways to the Grid middleware and application respectively.

\section{Conclusions}

This paper presents the Phosphorus control plane solution for Grid service enabled optical network infrastructure with the aim to facilitate user-controlled bandwidth provisioning for data-intensive and e-science applications. The Phosphorus solution, which consists in a new NCP that integrates a novel GNS provisioning mechanism and allows single-step reservation of network and Grid computing resources, is presented and discussed.

\section{Acknowledgements}

This work has been supported by European Commission through the IP PHOSPHORUS project.

\section{References}

[1] N. Ciulli et al., "The Grid-GMPLS Control Plane architecture," IST-PHOSPHORUS Deliverable 2.1, February 2007.

[2] G. Zervas et al., "Grid Optical User Network Interface (G.OUNI)," Open Grid Forum Draft, February 2007.

[3] ITU-T, "Architecture for the automatically switched optical network (ASON)", Recommendation G.8080/Y.1304, November 2001 (and Revision, January 2003).

[4] J. Zheng, B. Zhang, H.T. Mouftah, "Toward automated provisioning of advance reservation service in next-generation optical internet," IEEE Communications Magazine, Nov. 2006, pp. 68-74

[5] E. Mannie, “Generalized Multiprotocol Label Switching (GMPLS) Architecture,” IETF RFC3945, October 2004. 